\title{
Micromechanical Analysis of Thermo-Inelastic Multiphase Short-Fiber Composites
}

Jacob Aboudi

University of Virginia

Charlottesville, Virginia

March 1994

Prepared for

Lewis Research Center

Under Contract NAG3-1377

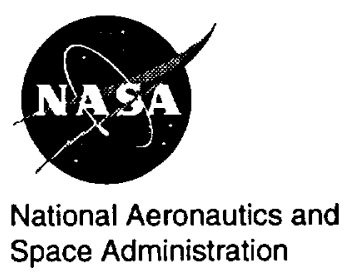

(NASA-CR-195290) MICROMECHANICAL

N94-30178

ANALYSIS OF THERMO-INELASTIC

MULTIPHASE SHORT-FIBER COMPOSITES

Final Report (Virginia Univ.)

$19 p$

Unclas 


\title{
Micromechanical Analysis of Thermo-Inelastic Multiphase Short-Fiber Composites
}

\author{
Jacob Aboudi* \\ University of Virginia \\ Department of Civil Engineering and Applied Mechanics \\ Charlottesville, VA 22903
}

\begin{abstract}
A micromechanical formulation is presented for the prediction of the overall thermoinelastic behavior of multiphase composites which consist of short fibers. The analysis is an extension of the generalized method of cells that was previously derived for inelastic composites with continuous fibers, and the reliability of which was critically examined in several situations. The resulting three-dimensional formulation is extremely general, wherein the analysis of thermo-inelastic composites with continuous fibers as well as particulate and porous inelastic materials are merely special cases.
\end{abstract}

\section{Introduction}

Modeling of the constitutive response of advanced composite materials continues to be an important part of these strategic materials' development. Analytical models that predict the effective behavior of composites are used not only by mechanicians in structural analysis of large-scale composites but also by materials scientists in developing new material systems. For an analytical model to fulfill these two distinct functions, it must be based on the micromechanics approach which allows one to generate the average response of a composite material from the properties of the individual constituents and their geometric arrangement. Only then can such a model be used by a material scientist to investigate the effect of different deformation mechanisms on the overall response of the composite in order to identify the appropriate constituents for a given application. At the same time, a micromechanical model, if it is to be used in a large-scale structural analysis program, must be computationally efficient in addition to being able to generate accurate displacement and stress fields at

\footnotetext{
*On leave from Tel-Aviv University, Israel
} 
both the macro- and micro-level. Knowledge of accurate field quantities at the micro-level is important in predicting failure of a structural element.

A number of models presently exist that can fulfill one or the other of the aforementioned tasks. On the other hand, there are very few working models that are both computationally efficient and sufficiently accurate at the micro- as well as the macro-level to satisfy the distinct needs of the material science and structural mechanics communities. One such model is the method of cells (Aboudi (1991)) and its generalization (Paley and Aboudi (1992), Aboudi (1993)) which is believed to have the potential of fulfilling both aforementioned tasks.

In the original formulation of the method of cells, a continuously-reinforced, unidirectional fibrous composite is modeled as a rectangular, doubly-periodic array of fibers embedded in a matrix phase. The periodic character of the assemblage allows one to identify a repeating unit cell that can be used as a building block to construct the entire composite. The properties of this repeating cell are thus representative of the properties of the entire assemblage. The unit cell consists of a single fiber subcell surrounded by three matrix subcells. Hence the name method of cells. The rectangular geometry of the repeating unit cell allows one to obtain an approximate solution for the stresses and strains in the individual subcells given some macroscopically homogeneous state of strain or stress applied to the composite. The approximate solution to the thus posed boundary-value problem is, in turn, used to determined macroscopic (average) or effective properties of the composite. The macroscopic behavior is displayed in terms of: effective elastic moduli; effective coefficients of thermal expansion; effective thermal conductivities; and effective stress-strain response in the inelastic region.

In the generalized method of cells for continuous fibrous composites, the repeating unit cell can consist of an arbitrary number of phases. Hence the generalized method of cells is capable of modeling a multiphase composite. This is of importance since even a two-phase composite with a metallic constituent behaves after the development of plasticity effects as a multiphase composite. A user's guide for a computer program based on the generalized method of cells has been recently presented by Aboudi and Pindera (1992).

In the original formulation of the method of cells for short-fiber composites, the unit cell consists of a single region occupied by the inclusion, surrounded by seven distinct matrix regions.

In the present paper the generalized method of cells is developed and formulated for the modeling of multiphase thermo-inelastic short-fiber composites. The presented threedimensional micromechanical analysis consists essentially of four steps. It starts by identifying a repeating volume element of the periodic multiphase composite, followed by the definition of macroscopic average stresses and strains from the microscopic ones. In the third step the continuity of tractions and displacements are imposed at the interfaces between the constituents. These establish, in conjunction with micro-equilibrium, the relationship between microscopic total, thermal and plastic strains and macroscopic strains via the relevant concentration tensors. In the final step the overall macroscopic constitutive equations of the composite are determined. These four steps form the basis of micro-to-macromechanics analyses which describe the behavior of heterogeneous media (Suquet, 1985). The resulting three-dimensional micromechanical analysis establishes the overall elasto-plastic behavior of the multiphase thermo-inelastic composites. This is expressed as a constitutive relation between the average stress, strain, thermal and plastic strains, in conjunction with the effective 
elastic stiffness tensor.

The micromechanically established constitutive equations are consistent in the sense that when they are employed to predict the effective coefficients of thermal expansion of the multiphase composite, they provide the same estimate obtained from Levin's result (1967). In the latter, the overall coefficient of thermal expansion is expressed in terms of the properties of the constituents in conjunction with the mechanical concentration factors. Similarly, the derived constitutive equations are also consistent in the sense that the resulting overall inelastic strain of the multiphase composite coincides with the expression obtained by Dvorak (1992). Here the overall inelastic strain is expressed in terms of the inelastic strains of the phases in conjunction with the mechanical concentration factors. Both Levin's and Dvorak's results follow from the relationship between the overall and local transformation fields which can be established by invoking the reciprocal theorem (Dvorak and Benveniste (1992)).

\section{Model Description}

Consider a composite material with a periodic structure whose repeating volume element consists of $N_{\alpha} \times N_{\beta} \times N_{\gamma}$ rectangular parallelepiped subcells. The volume of each one of the subcells is $d_{\alpha} h_{\beta} \ell_{\gamma}$, where $\alpha, \beta$ and $\gamma$ are running indices: $\alpha=1, \ldots, N_{\alpha} ; \beta=1, \ldots N_{\beta}$ $; \gamma=1, \ldots, N_{\gamma}$, in the $x_{1}, x_{2}$ and $x_{3}$-directions, respectively. The total volume of the repeating volume element is $d h \ell$ where $d=\sum_{\alpha=1}^{N_{\alpha}} d_{\alpha}, h=\sum_{\beta=1}^{N_{\beta}} h_{\beta}, \ell=\sum_{\ell=1}^{N_{\gamma}} \ell_{\gamma}$. In Fig. 1 an example is shown for a repeating volume element with $N_{\alpha}=3, N_{\beta}=4$ and $N_{\gamma}=2$.

Each of the subcells can be filled, in general, by an elastic-viscoplastic temperature dependent material. Consequently, the overall behavior of the composite should simulate a multiphase thermo-viscoplastic composite. By appropriately selecting the subcells dimensions or, alternatively, by appropriately filling the subcells, various types of composites can be considered. Thus, unidirectional long-fiber composites, short-fiber composites, porous materials and laminated materials can be modeled as special cases. Consequently, the present micromechanical multiphase composite model is very general and various situations can be obtained as special cases.

By an approximate micromechanical analysis of the detailed interactions of the subcells of the repeating volume element, overall constitutive relations which govern the effective behavior of the multiphase viscoelastic composite, can be established. This analysis relies on the requirements that static equilibrium of the materials in the subcells are ensured, and that continuity of the displacements and tractions between neighboring subcells within the repeating volume element, as well as between neighboring repeating volume elements is satisfied on an average basis.

Let us introduce local coordinates $\left(\bar{x}_{1}^{(\alpha)}, \bar{x}_{2}^{(\beta)}, \bar{x}_{3}^{(\gamma)}\right)$ whose origin is located at the center of the subcell $(\alpha \beta \gamma)$. These local coordinates are shown in Fig. 2 for subcell $(\alpha \beta \gamma)$ and the neighboring one $(\hat{\alpha} \gamma \beta)$ in the $x_{1}$ - direction, where $\hat{\alpha}$ is defined to have the form

$$
\hat{\alpha}= \begin{cases}\alpha+1 & \alpha<N_{\alpha} \\ 1 & \alpha=N_{\alpha}\end{cases}
$$

This definition ensures that for $\alpha<N_{\alpha}$ the neighboring subcell in the $x_{1}$ - direction is the one labelled by $(\alpha+1, \beta, \gamma)$ within the repeating volume element, whereas for $\alpha=N_{\alpha}$ 
the neighboring subcell is within the next repeating volume element whose first subcell is $(1 \beta \gamma)$. Similarly, $\hat{\beta}$ and $\hat{\gamma}$ are defined by

$$
\begin{gathered}
\hat{\beta}= \begin{cases}\beta+1 & \beta<N_{\beta} \\
1 & \beta=N_{\beta}\end{cases} \\
\hat{\gamma}= \begin{cases}\gamma+1 & \gamma<N_{\gamma} \\
1 & \gamma=N_{\gamma}\end{cases}
\end{gathered}
$$

Since the average behavior of the composite is sought, it is sufficient to consider a first order theory in which the displacements $u_{i}^{(\alpha \beta \gamma)}$ in the subcell are expanded linearly in terms of the distances from the center of the subcell, i.e., in terms of $\bar{x}_{1}^{(\alpha)}, \bar{x}_{2}^{(\beta)}$, and $\bar{x}_{3}^{(\gamma)}$. Thus, the following first order expansion in the subcell $(\alpha \beta \gamma)$ is considered

$$
u_{i}^{(\alpha \beta \gamma)}=w_{i}^{(\alpha \beta \gamma)}+\bar{x}_{1}^{(\alpha)} \phi_{i}^{(\alpha \beta \gamma)}+\bar{x}_{2}^{(\beta)} \chi_{i}^{(\alpha \beta \gamma)}+\bar{x}_{3}^{(\gamma)} \psi_{i}^{(\alpha \beta \gamma)} \quad i=1,2,3
$$

where $w_{i}^{(\alpha \beta \gamma)}$ are the displacement components at the center of the subcell, and $\phi_{i}^{(\alpha \beta \gamma)}, \chi_{i}^{(\alpha \beta \gamma)}$ and $\psi_{i}^{(\alpha \beta \gamma)}$ are microvariables that characterize the linear dependence of the displacement $u_{i}^{(\alpha \beta \gamma)}$ on the local coordinates $\bar{x}_{1}^{(\alpha)}, \bar{x}_{2}^{(\beta)}, \bar{x}_{3}^{(\gamma)}$. In eqn. (4) and the sequel, repeated Greek letters do not imply summation. Note that due to the linearity of eqn. (4), static equilibrium of the material within the subcell $(\alpha \beta \gamma)$ is ensured.

The components of the small strain tensor are given by

$$
\epsilon_{i j}^{(\alpha \beta \gamma)}=\frac{1}{2}\left(\partial_{i} u_{j}^{(\alpha \beta \gamma)}+\partial_{j} u_{i}^{(\alpha \beta \gamma)}\right) \quad i, j=1,2,3
$$

where $\partial_{1}=\partial / \partial \bar{x}_{1}^{(\alpha)}, \partial_{2}=\partial / \partial \bar{x}_{2}^{(\beta)}$ and $\partial_{3}=\partial / \partial \bar{x}_{3}^{(\gamma)}$.

The average strains in the subcell, $\bar{\epsilon}_{i j}^{(\alpha \beta \gamma)}$ are given according to eqns. (4)-(5) by

$$
\begin{aligned}
\bar{\epsilon}_{11}^{(\alpha \beta \gamma)} & =\phi_{1}^{(\alpha \beta \gamma)}, \bar{\epsilon}_{22}^{(\alpha \beta \gamma)}=\chi_{2}^{(\alpha \beta \gamma)}, \bar{\epsilon}_{33}^{(\alpha \beta \gamma)}=\psi_{3}^{(\alpha \beta \gamma)} \\
2 \bar{\epsilon}_{23}^{(\alpha \beta \gamma)} & =\chi_{3}^{(\alpha \beta \gamma)}+\psi_{2}^{(\alpha \beta \gamma)} \\
2 \bar{\epsilon}_{13}^{(\alpha \beta \gamma)} & =\psi_{1}^{(\alpha \beta \gamma)}+\phi_{3}^{(\alpha \beta \gamma)} \\
2 \bar{\epsilon}_{12}^{(\alpha \beta \gamma)} & =\chi_{1}^{(\alpha \beta \gamma)}+\phi_{2}^{(\alpha \beta \gamma)}
\end{aligned}
$$

The average strains in the composite are expressed as

$$
\bar{\epsilon}_{i j}=\frac{1}{d h \ell} \sum_{\alpha=1}^{N_{\mathrm{a}}} \sum_{\beta=1}^{N_{\beta}} \sum_{\gamma=1}^{N_{\gamma}} d_{\alpha} h_{\beta} \ell_{\gamma} \bar{\epsilon}_{i j}^{(\alpha \beta \gamma)}
$$

The constitutive equation of the elastic-viscoplastic temperature-dependent material that occupies subcell $(\alpha \beta \gamma)$ is expressed as a relationship between the averages of stress $\bar{\sigma}_{i j}^{(\alpha \beta \gamma)}$, total strain $\bar{\epsilon}_{i j}^{(\alpha \beta \gamma)}$, inelastic strain $\bar{\epsilon}_{i j}^{I(\alpha \beta \gamma)}$, and the thermal strain $\bar{\epsilon}_{i j}^{T(\alpha \beta \gamma)}=\alpha_{i j}^{(\alpha \beta \gamma)} \Delta T$ ( where $\alpha_{i j}^{(\alpha \beta \gamma)}$ is the coefficient of thermal expansion tensor, and $\Delta T$ is the temperature deviation $T-T_{R}$ from a reference temperature $T_{R}$ ), i.e.

$$
\bar{\sigma}_{i j}^{(\alpha \beta \gamma)}=C_{i j k \ell}^{(\alpha \beta \gamma)}\left(\bar{\epsilon}_{k \ell}^{(\alpha \beta \gamma)}-\bar{\epsilon}_{k \ell}^{I(\alpha \beta \gamma)}-\bar{\epsilon}_{k \ell}^{T(\alpha \beta \gamma)}\right)
$$


where $C_{i j k \ell}^{(\alpha \beta \gamma)}$ is the elastic stiffness tensor of the material.

The average stress in the composite is determined from

$$
\bar{\sigma}_{i j}=\frac{1}{d h \ell} \sum_{\alpha=1}^{N_{\alpha}} \sum_{\beta=1}^{N_{\beta}} \sum_{\gamma=1}^{N_{\gamma}} d_{\alpha} h_{\beta} \ell_{\gamma} \bar{\sigma}_{i j}^{(\alpha \beta \gamma)}
$$

It will be shown in the following that by employing the displacement and traction continuity conditions at the interfaces between the subcells of the repeating volume element, and at the interfaces between neighboring repeating volume elements, it is possible to eliminate the microvariables and obtain, via a smoothing operation (homogenization), a set of continuum equations that model the overall behavior of the multiphase short-fiber composite. This is achieved by establishing relationships which connect the microstrains at the subcells to the total overall macrostrains in the composite via the appropriate concentration tensors.

\section{Interfacial Continuity of Displacements}

At any instant, the displacement components must be continuous at the various interfaces of the repeating unit cell, and at the interfaces between neighboring repeating cells. This implies that for $\alpha=1, \ldots, N_{\alpha} ; \beta=1, \ldots, N_{\beta} ; \gamma=1, \ldots, N_{\gamma}$, the following relations hold

$$
\begin{aligned}
& u_{i}^{(\alpha \beta \gamma)} \mid \begin{array}{ll}
\bar{x}_{1}^{(\alpha)}=\frac{d_{\alpha}}{2} & u_{i}^{(\hat{\alpha} \beta \gamma)} \mid \bar{x}_{1}^{(\hat{\alpha})}=\frac{-d_{\dot{\alpha}}}{2} \\
u_{i}^{(\alpha \beta \gamma)} \mid \bar{x}_{2}^{(\beta)}=\frac{h_{\beta}}{2} & u_{i}^{(\alpha \hat{\beta} \gamma)} \mid \bar{x}_{2}^{(\widehat{\beta})}=\frac{-h_{\widehat{\beta}}}{2} \\
u_{i}^{(\alpha \beta \gamma)} \mid \bar{x}_{3}^{(\gamma)}=\frac{l_{\gamma}}{2} & u_{i}^{(\alpha \beta \hat{\gamma})} \mid \bar{x}_{3}^{(\hat{\gamma})}=\frac{-l_{\widehat{\gamma}}}{2}
\end{array}
\end{aligned}
$$

Notice that with the definition of $\hat{\alpha}, \hat{\beta}$ and $\hat{\gamma}$, eqns.(1)- (3), continuity of the displacement at the interfaces between neighboring repeating cells is ensured.

Continuity conditions (10) - (12) are imposed at the interfaces in an average sense. For example, eqn. (10) is applied in the form

$$
\begin{gathered}
\int_{-h_{\beta} / 2}^{h_{\beta} / 2} \int_{-\ell_{\gamma} / 2}^{\ell_{\gamma} / 2}\left[u_{i}^{(\alpha \beta \gamma)} \mid \bar{x}_{1}^{(\alpha)}=\frac{d_{\alpha}}{2}\right] d \bar{x}_{2}^{(\beta)} d \bar{x}_{3}^{(\gamma)}= \\
\int_{-h_{\beta} / 2}^{h_{\beta} / 2} \int_{-\ell_{\gamma} / 2}^{\ell_{\gamma} / 2}\left[u_{i}^{(\hat{\alpha} \beta \gamma)} \mid \bar{x}_{1}^{(\hat{\alpha})}=\frac{-d_{\alpha}}{2}\right] d \bar{x}_{2}^{(\beta)} d \bar{x}_{3}^{(\gamma)}
\end{gathered}
$$

Using eqn. (4) in (10) one obtains

$$
w_{i}^{(\alpha \beta \gamma)}+\frac{1}{2} d_{\alpha} \phi_{i}^{(\alpha \beta \gamma)}=w^{(\hat{\alpha} \beta \gamma)}-\frac{1}{2} d_{\widehat{\alpha}} \phi_{i}^{(\hat{\alpha} \beta \gamma)}
$$


Two similar equations would result from the use of eqn. (4) in (11) and (12):

$$
\begin{aligned}
& w_{i}^{(\alpha \beta \gamma)}+\frac{1}{2} h_{\beta} \chi_{i}^{(\alpha \beta \gamma)}=w_{i}^{(\alpha \hat{\beta} \gamma)}-\frac{1}{2} h_{\hat{\beta}} \chi_{i}^{(\alpha \hat{\beta} \gamma)} \\
& w_{i}^{(\alpha \beta \gamma)}+\frac{1}{2} \ell_{\gamma} \psi_{i}^{(\alpha \beta \gamma)}=w_{i}^{(\alpha \beta \hat{\gamma})}-\frac{1}{2} \ell_{\hat{\gamma}} \psi_{i}^{(\alpha \beta \hat{\gamma})}
\end{aligned}
$$

All field variables in eqn. (14) are evaluated at the center line $x_{1}^{(\alpha)}$ of the subcell $(\alpha \beta \gamma)$, and at the center line $x_{1}^{(\hat{\alpha})}$ of the subcell $(\hat{\alpha} \beta \gamma)$, see Fig. 2. In the final form of the displacement continuity relations it is required to evaluate all terms at a common location. To this end, let $x_{1}^{(I)}$ denote the location of the interface between subcell $(\alpha \beta \gamma)$ and the following one $(\hat{\alpha} \beta \gamma)$. It follows that

$$
x_{1}^{(\alpha)}=x_{1}^{(I)}-\frac{1}{2} d_{\alpha}
$$

In addition, the location of the center line $x_{1}^{(\hat{\alpha})}$ of the subcell $(\hat{\alpha} \beta \gamma)$ with respect to $x_{1}^{(I)}$ is given by

$$
x_{1}^{(\widehat{\alpha})}=x_{1}^{(I)}+\frac{1}{2} d_{\widehat{\alpha}}
$$

By expanding the field variables in eqn. (14) in Taylor's series about the common interface $x_{1}^{(I)}$ and dropping second order terms, we obtain

$$
w_{i}^{(\alpha \beta \gamma)}-\frac{1}{2} d_{\alpha}\left(\frac{\partial}{\partial x_{1}} w_{i}^{(\alpha \beta \gamma)}-\phi_{i}^{(\alpha \beta \gamma)}\right)=w_{i}^{(\hat{\alpha} \beta \gamma)}+\frac{1}{2} d_{\widehat{\alpha}}\left(\frac{\partial}{\partial x_{1}} w_{i}^{(\hat{\alpha} \beta \gamma)}-\phi_{i}^{(\hat{\alpha} \beta \gamma)}\right)
$$

where all field variables in (19) are evaluated at the common interface $x_{1}^{(I)}$. The discrete structure of the composite is eliminated by a smoothing operation according to which eqn. (19) is assumed to be valid at any point of the homogenized medium, which effectively represents the multiphase composite, for all $\alpha, \beta$ and $\gamma$. For a composite subjected to homogeneous boundary conditions, the behavior of all repeating cells is identical and a uniform field exists at the equivalent homogeneous medium.

Let $F_{i}^{(\alpha)}$ be defined as:

$$
F_{i}^{(\alpha)}=w_{i}^{(\alpha \beta \gamma)}+f_{i}^{(\alpha)}-w_{i}^{(\hat{\alpha} \beta \gamma)}+f_{i}^{(\widehat{\alpha})}
$$

where

$$
f_{i}^{(\alpha)}=-\frac{1}{2} d_{\alpha}\left(\frac{\partial}{\partial x_{1}} w_{i}^{(\alpha \beta \gamma)}-\phi_{i}^{(\alpha \beta \gamma)}\right)
$$

Consequently, equations (19) can be rewritten as

$$
F_{i}^{(\alpha)}=0 \quad, \quad \alpha=1, \ldots, N_{\alpha}
$$

A similar derivation based on eqns. (15) -(16) would respectively provide the following two relations 


$$
G_{i}^{(\beta)}=0 \quad, \quad \beta=1, \ldots, N_{\beta}
$$

and

$$
H_{i}^{(\gamma)}=0 \quad, \quad \gamma=1, \ldots, N_{\gamma}
$$

where the following definitions have been employed

$$
\begin{aligned}
G_{i}^{(\beta)} & =w_{i}^{(\alpha \beta \gamma)}+g_{i}^{(\beta)}-w_{i}^{(\alpha \hat{\beta} \gamma)}+g_{i}^{(\widehat{\beta})} \\
H_{i}^{(\gamma)} & =w_{i}^{(\alpha \beta \gamma)}+h_{i}^{(\gamma)}-w_{i}^{(\alpha \beta \widehat{\gamma})}+h_{i}^{(\hat{\gamma})} \\
g_{i}^{(\beta)} & =-\frac{1}{2} h_{\beta}\left(\frac{\partial}{\partial x_{2}} w_{i}^{(\alpha \beta \gamma)}-\chi_{i}^{(\alpha \beta \gamma)}\right) \\
h_{i}^{(\gamma)} & =-\frac{1}{2} \ell_{\gamma}\left(\frac{\partial}{\partial x_{3}} w_{i}^{(\alpha \beta \gamma)}-\psi_{i}^{(\alpha \beta \gamma)}\right)
\end{aligned}
$$

Like eqn. (22), eqns. (23)-(24) are simultaneously valid at any point of the homogenized medium, which effectively represents the multiphase composite subjected to homogeneous boundary conditions, for all $\alpha, \beta$ and $\gamma$.

From eqns. (22) - (24) we obtain, respectively, that

$$
\sum_{\alpha=1}^{N_{\alpha}} F_{i}^{(\alpha)}=0 \quad, \quad \sum_{\beta=1}^{N_{\beta}} G_{i}^{(\beta)}=0 \quad, \quad \sum_{\gamma=1}^{N_{\gamma}} H_{i}^{(\gamma)}=0
$$

The three summations provide, respectively, that

$$
\sum_{\alpha=1}^{N_{\alpha}} f_{i}^{(\alpha)}=0 \quad, \quad \sum_{\beta=1}^{N_{\beta}} g_{i}^{(\beta)}=0 \quad, \quad \sum_{\gamma=1}^{N_{\gamma}} h_{i}^{(\gamma)}=0
$$

Since

$$
\frac{\partial}{\partial x_{1}} f_{i}^{(\alpha)}=0 \quad, \quad \frac{\partial}{\partial x_{2}} g_{i}^{(\beta)}=0 \quad, \quad \frac{\partial}{\partial x_{3}} h_{i}^{(\gamma)}=0
$$

for all $\alpha, \beta$ and $\gamma$, it follows by differentiation of eqns. (22), (23) and (24) with respect to $x_{1}, x_{2}$ and $x_{3}$, respectively, that

$$
\begin{aligned}
& \frac{\partial}{\partial x_{1}} w_{i}^{(\alpha \beta \gamma)}=\frac{\partial}{\partial x_{1}} w_{i}^{(\hat{\alpha} \beta \gamma)} \\
& \frac{\partial}{\partial x_{2}} w_{i}^{(\alpha \beta \gamma)}=\frac{\partial}{\partial x_{2}} w_{i}^{(\alpha \hat{\beta} \gamma)} \\
& \frac{\partial}{\partial x_{3}} w_{i}^{(\alpha \beta \gamma)}=\frac{\partial}{\partial x_{3}} w_{i}^{(\alpha \beta \widehat{\gamma})}
\end{aligned}
$$


Equations (31) are satisfied by assuming that common displacement functions, $w_{i}$, exist such that

$$
w_{i}^{(\alpha \beta \gamma)}=w_{i}
$$

for all $\alpha, \beta$ and $\gamma$. This assumption is consistent with the fact that the entire repeating volume element of the periodic composite is mapped by a smoothing operation into a single point within the equivalent continuum medium. The displacement components at this point are $w_{i}$.

Using eqns. (33), we readily obtain from (30) the following set of continuum relations

$$
\begin{aligned}
& \sum_{\alpha=1}^{N_{a}} d_{\alpha} \phi_{i}^{(\alpha \beta \gamma)}=d \frac{\partial}{\partial x_{1}} w_{i} \quad, \quad \begin{array}{l}
\beta=1, \ldots, N_{\beta} \\
\gamma=1, \ldots, N_{\gamma}
\end{array} \\
& \sum_{\beta=1}^{N_{\beta}} h_{\beta} \chi_{i}^{(\alpha \beta \gamma)}=h \frac{\partial}{\partial x_{2}} w_{i} \quad, \quad \begin{array}{l}
\alpha=1, \ldots, N_{\alpha} \\
\gamma=1, \ldots, N_{\gamma}
\end{array} \\
& \sum_{\gamma=1}^{N_{\gamma}} \ell_{\gamma} \psi_{i}^{(\alpha \beta \gamma)}=\ell \frac{\partial}{\partial x_{3}} w_{i} \quad, \quad \begin{array}{l}
\alpha=1, \ldots, N_{\alpha} \\
\beta=1, \ldots, N_{\beta}
\end{array}
\end{aligned}
$$

Continuum relations (34)-(36) are expressed in terms of the microvariables $\phi_{i}^{(\alpha \beta \gamma)}, \chi_{i}^{(\alpha \beta \gamma)}$, $\psi_{i}^{(\alpha \beta \gamma)}$. It is possible to derive an equivalent system of equations which are expressed in terms of the average subcell strains $\bar{\epsilon}_{i j}^{(\alpha \beta \gamma)}$.

The average composite strains, $\bar{\epsilon}_{i j}$, were defined in eqn. (7). Let us first show that

$$
\bar{\epsilon}_{i j}=\frac{1}{2}\left(\frac{\partial w_{i}}{\partial x_{j}}+\frac{\partial w_{j}}{\partial x_{i}}\right)
$$

For $i=j=1$, let us multiply eqn. (34) by $h_{\beta} \ell_{\gamma}$ and perform a summation over $\beta$ from 1 to $N_{\beta}$, and over $\gamma$ from 1 to $N_{\gamma}$. This provides

$$
\sum_{\alpha=1}^{N_{a}} \sum_{\beta=1}^{N_{\beta}} \sum_{\gamma=1}^{N_{\gamma}} d_{\alpha} h_{\beta} \ell_{\gamma} \phi_{1}^{(\alpha \beta \gamma)}=d h \ell \frac{\partial w_{1}}{\partial x_{1}}
$$

Comparing eqn. (38) with (7) and using the first equality in (6), gives $\bar{\epsilon}_{11}=\frac{\partial w_{1}}{\partial x_{1}}$.

For $i=1, j=2$ let us multiply eqn. (34) with $i=2$ by $h_{\beta} \ell_{\gamma}$ and perform a summation over $\beta$ from 1 to $N_{\beta}$, and over $\gamma$ from 1 to $N_{\gamma}$. Similarly, let us multiply eqn. (35) with $i=1$ by $d_{\alpha} \ell_{\gamma}$ and perform summations over all $\alpha$ and $\gamma$. By adding the resulting relations we obtain

$$
\sum_{\alpha, \beta, \gamma} d_{\alpha} h_{\beta} \ell_{\gamma}\left(\phi_{2}^{(\alpha \beta \gamma)}+\chi_{1}^{(\alpha \beta \gamma)}\right)=d h \ell\left(\frac{\partial w_{2}}{\partial x_{1}}+\frac{\partial w_{1}}{\partial x_{2}}\right)
$$

Again, using the last equality in (6) in conjunction with equation (7) and comparing this to the above expression gives $\bar{\epsilon}_{12}=\left(\frac{\partial w_{2}}{\partial x_{1}}+\frac{\partial w_{1}}{\partial x_{2}}\right) / 2$. In a similar manar the other four relations in (37) can be established. 
It is now possible to express the continuum eqns. (34)-(36) in terms of $\bar{\epsilon}_{i j}^{(\alpha \beta \gamma)}$ and $\bar{\epsilon}_{i j}$. Setting $i=1,2$ and 3 in (34)-(36), respectively, we obtain

$$
\begin{aligned}
& \sum_{\alpha=1}^{N_{\alpha}} d_{\alpha} \bar{\epsilon}_{11}^{(\alpha \beta \gamma)}=d \bar{\epsilon}_{11} \quad, \quad \begin{array}{l}
\beta=1, \ldots, N_{\beta} \\
\gamma=1, \ldots, N_{\gamma}
\end{array} \\
& \sum_{\beta=1}^{N_{\beta}} h_{\beta} \bar{\epsilon}_{22}^{(\alpha \beta \gamma)}=h \bar{\epsilon}_{22} \quad, \quad \begin{array}{l}
\alpha=1, \ldots, N_{\alpha} \\
\gamma=1, \ldots, N_{\gamma}
\end{array} \\
& \sum_{\gamma=1}^{N_{\gamma}} \ell_{\gamma} \bar{\epsilon}_{33}^{(\alpha \beta \gamma)}=\ell \bar{\epsilon}_{33} \quad, \quad \begin{array}{l}
\alpha=1, \ldots, N_{\alpha} \\
\beta=1, \ldots, N_{\beta}
\end{array}
\end{aligned}
$$

The addition of eqn. (34) (with $i=2$ ) multiplied by $h_{\beta}$ and summed over all $\beta$, to (35) (with $i=1$ ) multiplied by $d_{\alpha}$ and summed over all $\alpha$, yields

$$
\sum_{\alpha=1}^{N_{\alpha}} \sum_{\beta=1}^{N_{\beta}} d_{\alpha} h_{\beta}\left(\phi_{2}^{(\alpha \beta \gamma)}+\chi_{1}^{(\alpha \beta \gamma)}\right)=d h\left(\frac{\partial w_{2}}{\partial x_{1}}+\frac{\partial w_{1}}{\partial x_{2}}\right)
$$

i.e.,

$$
\sum_{\alpha=1}^{N_{\alpha}} \sum_{\beta=1}^{N_{\beta}} d_{\alpha} h_{\beta} \bar{\epsilon}_{12}^{(\alpha \beta \gamma)}=d h \bar{\epsilon}_{12} \quad, \quad \gamma=1, \ldots, N_{\gamma}
$$

which are the desired continuum equations expressed in terms of subcell and average strains. Similar operations give

$$
\begin{array}{ll}
\sum_{\beta=1}^{N_{\beta}} \sum_{\gamma=1}^{N_{\gamma}} h_{\beta} \ell_{\gamma} \bar{\epsilon}_{23}^{(\alpha \beta \gamma)}=h \ell \bar{\epsilon}_{23} \quad, \quad \alpha=1, \ldots, N_{\alpha} \\
\sum_{\alpha=1}^{N_{\alpha}} \sum_{\gamma=1}^{N_{\gamma}} d_{\alpha} \ell_{\gamma} \bar{\epsilon}_{13}^{(\alpha \beta \gamma)}=d \ell \bar{\epsilon}_{13} \quad, \quad \beta=1, \ldots, N_{\beta}
\end{array}
$$

Equations (40) - (45), form a set of $N_{\alpha} N_{\beta}+N_{\beta} N_{\gamma}+N_{\alpha} N_{\gamma}+N_{\alpha}+N_{\beta}+N_{\gamma}$ relations which replace eqns. (34)-(36). These relations can be written in a matrix form as follows

$$
\boldsymbol{A}_{G} \boldsymbol{\epsilon}_{\boldsymbol{s}}=\boldsymbol{J} \overline{\boldsymbol{\epsilon}}
$$

where the 6-order average strain-rate vector is defined by

$$
\bar{\epsilon}=\left(\bar{\epsilon}_{11}, \bar{\epsilon}_{22}, \bar{\epsilon}_{33}, 2 \bar{\epsilon}_{23}, 2 \bar{\epsilon}_{13}, 2 \bar{\epsilon}_{12}\right)
$$

and the $6 N_{\alpha} N_{\beta} N_{\gamma}$ order subcell strain vector, $\epsilon_{s}$, is defined by

$$
\epsilon_{s}=\left(\bar{\epsilon}^{(111)}, \ldots, \bar{\epsilon}^{\left(N_{\alpha} N_{\beta} N_{\gamma}\right)}\right),
$$

where the 6 components of the vector $\overline{\boldsymbol{\epsilon}}^{(\alpha \beta \gamma)}$ are arranged as in eqn. (47). The matrix $\boldsymbol{A}_{G}$ is $N_{\alpha}\left(N_{\beta}+N_{\gamma}+1\right)+N_{\beta}\left(N_{\gamma}+1\right)+N_{\gamma}$ by $6 N_{\alpha} N_{\beta} N_{\gamma}$, while $\boldsymbol{J}$ is a $N_{\alpha}\left(N_{\beta}+N_{\gamma}+1\right)+N_{\beta}\left(N_{\gamma}+1\right)+N_{\gamma}$ by 6 matrix. It should be noted that the matrix $\boldsymbol{A}_{G}$ involves the geometrical dimensions of the repeating cell only. 


\section{Interfacial Continuity of Tractions}

The tractions must be continuous at the interfaces between the subcells of the repeating cells, and at the interfaces between neighboring repeating cells. These conditions, imposed in an average sense, give

$$
\begin{aligned}
& \bar{\sigma}_{1 i}^{(\alpha \beta \gamma)}=\bar{\sigma}_{1 i}^{(\hat{\alpha} \beta \gamma)} \\
& \bar{\sigma}_{2 i}^{(\alpha \beta \gamma)}=\bar{\sigma}_{2 i}^{(\alpha \hat{\beta} \gamma)} \\
& \bar{\sigma}_{3 i}^{(\alpha \beta \gamma)}=\bar{\sigma}_{3 i}^{(\alpha \beta \hat{\gamma})}
\end{aligned}
$$

where $i=1,2,3 ; \alpha=1, \ldots, N_{\alpha} ; \beta=1, \ldots, N_{\beta}$ and $\gamma=1, \ldots, N_{\gamma}$. It can be easily verified that these equations involve some repetitions. It can be shown that the system of independent interfacial conditions is

$$
\begin{aligned}
& \bar{\sigma}_{11}^{(\alpha \beta \gamma)}=\bar{\sigma}_{11}^{(\hat{\alpha} \beta \gamma)} \\
& \alpha=1, \ldots, N_{\alpha}-1 \\
& \beta=1, \ldots, N_{\beta} \\
& \gamma=1, \ldots, N_{\gamma} \\
& \bar{\sigma}_{22}^{(\alpha \beta \gamma)}=\bar{\sigma}_{22}^{(\alpha \hat{\beta} \gamma)} \\
& \alpha=1, \ldots, N_{\alpha} \\
& \beta=1, \ldots, N_{\beta}-1 \\
& \gamma=1, \ldots, N_{\gamma} \\
& \bar{\sigma}_{33}^{(\alpha \beta \gamma)}=\bar{\sigma}_{33}^{(\alpha \beta \widehat{\gamma})} \\
& \alpha=1, \ldots, N_{\alpha} \\
& \beta=1, \ldots, N_{\beta} \\
& \gamma=1, \ldots, N_{\gamma}-1 \\
& \bar{\sigma}_{23}^{(\alpha \beta \gamma)}=\bar{\sigma}_{23}^{\left(\alpha \widehat{\beta}_{\gamma}\right)} \\
& \alpha=1, \ldots, N_{\alpha} \\
& \beta=1, \ldots, N_{\beta}-1 \\
& \gamma=1, \ldots, N_{\gamma} \\
& \bar{\sigma}_{32}^{(\alpha \beta \gamma)}=\bar{\sigma}_{32}^{(\alpha \beta \hat{\gamma})} \\
& \alpha=1, \ldots, N_{\alpha} \\
& \beta=N_{\beta} \\
& \gamma=1, \ldots, N_{\gamma}-1
\end{aligned}
$$




$$
\begin{aligned}
& \bar{\sigma}_{13}^{(\alpha \beta \gamma)}=\bar{\sigma}_{13}^{(\hat{\alpha} \beta \gamma)} \quad \alpha=1, \ldots, N_{\alpha}-1 \\
& \beta=1, \ldots, N_{\beta} \\
& \gamma=1, \ldots, N_{\gamma} \\
& \bar{\sigma}_{31}^{(\alpha \beta \gamma)}=\bar{\sigma}_{31}^{(\alpha \beta \hat{\gamma})} \quad \alpha=N_{\alpha} \\
& \beta=1, \ldots, N_{\beta} \\
& \gamma=1, \ldots, N_{\gamma}-1 \\
& \bar{\sigma}_{12}^{(\alpha \beta \gamma)}=\bar{\sigma}_{12}^{(\hat{\alpha} \beta \gamma)} \quad \alpha=1, \ldots, N_{\alpha}-1 \\
& \beta=1, \ldots, N_{\beta} \\
& \gamma=1, \ldots, N_{\gamma} \\
& \bar{\sigma}_{21}^{(\alpha \beta \gamma)}=\bar{\sigma}_{21}^{(\alpha \hat{\beta} \gamma)} \\
& \alpha=N_{\alpha} \\
& \beta=1, \ldots, N_{\beta}-1 \\
& \gamma=1, \ldots, N_{\gamma}
\end{aligned}
$$

As in the case of the displacement continuity conditions, the continuum model requires that for a composite subjected to homogeneous boundary conditions, conditions (50) - (58) are satisfied at all points of the homogeneous equivalent medium. By using the constitutive laws (8), it is possible to represent eqns. (50)-(58) in matrix form, that is,

$$
A_{M}\left(\epsilon_{s}-\epsilon_{s}^{I}-\epsilon_{s}^{T}\right)=0
$$

where the $6 N_{\alpha} N_{\beta} N_{\gamma}-\left(N_{\alpha} N_{\beta}+N_{\alpha} N_{\gamma}+N_{\beta} N_{\gamma}\right)-\left(N_{\alpha}+N_{\beta}+N_{\gamma}\right)$ by $6 N_{\alpha} N_{\beta} N_{\gamma}$ matrix $\boldsymbol{A}_{M}$ involves the elastic properties $C^{(\alpha \beta \gamma)}$ of material in the subcell, and

$$
\begin{aligned}
\epsilon_{s}^{I} & =\left(\bar{\epsilon}^{I(111)}, \ldots, \bar{\epsilon}^{I\left(N_{\alpha} N_{\beta} N_{\gamma}\right)}\right) \\
\epsilon_{s}^{T} & =\left(\bar{\epsilon}^{T(111)}, \ldots, \bar{\epsilon}^{T\left(N_{\alpha} N_{\beta} N_{\gamma}\right)}\right)
\end{aligned}
$$

\section{Overall Inelastic Thermomechanical Constitutive Law}

The combination of equation (59) and (46) leads to

$$
\tilde{A} \epsilon_{s}-\tilde{D}\left(\epsilon_{s}^{I}+\epsilon_{s}^{T}\right)=K \bar{\epsilon}
$$

where 


$$
\tilde{\boldsymbol{A}}=\left[\begin{array}{c}
\boldsymbol{A}_{M} \\
\boldsymbol{A}_{G}
\end{array}\right] \quad, \quad \tilde{D}=\left[\begin{array}{c}
\boldsymbol{A}_{M} \\
\mathbf{0}
\end{array}\right] \quad, \quad \boldsymbol{K}=\left[\begin{array}{l}
\mathbf{0} \\
\boldsymbol{J}
\end{array}\right]
$$

Solving eqn. (62) for the subcell strains $\epsilon_{s}$ yields

$$
\epsilon_{s}=A \bar{\epsilon}+D\left(\epsilon_{s}^{I}+\epsilon_{s}^{T}\right)
$$

where

$$
\boldsymbol{A}=\tilde{\boldsymbol{A}}^{-1} \boldsymbol{K} \quad, \quad \boldsymbol{D}=\tilde{\boldsymbol{A}}^{-1} \tilde{\boldsymbol{D}}
$$

Let the mechanical concentration matrix $A$ be partitioned into $N_{\alpha} N_{\beta} N_{\gamma} 6$-order square submatrices in the form

$$
\boldsymbol{A}=\left[\begin{array}{c}
\boldsymbol{A}^{(111)} \\
\vdots \\
\boldsymbol{A}^{\left(N_{\mathrm{a}} N_{\beta} N_{\gamma}\right)}
\end{array}\right]
$$

Similarly, let

$$
\boldsymbol{D}=\left[\begin{array}{c}
\boldsymbol{D}^{(111)} \\
\vdots \\
\boldsymbol{D}^{\left(N_{\alpha} N_{\beta} N_{\gamma}\right)}
\end{array}\right]
$$

where $D^{(\alpha \beta \gamma)}$ are square matrices of $6 N_{\alpha} N_{\beta} N_{\gamma}$ order.

It follows from eqn. (63) that

$$
\overline{\boldsymbol{\epsilon}}^{(\alpha \beta \gamma)}=A^{(\alpha \beta \gamma)} \overline{\boldsymbol{\epsilon}}+D^{(\alpha \beta \gamma)}\left(\epsilon_{s}^{I}+\epsilon_{s}^{T}\right)
$$

Equation (66) expresses the average strain in the subcell in terms of the uniform overall strain $\bar{\epsilon}$ (the applied macrostrain) and the subcell inelastic and thermal strains, via the concentration matrices $\boldsymbol{A}^{(\alpha \beta \gamma)}$ and $D^{(\alpha \beta \gamma)}$. Notice that the same matrices $D^{(\alpha \beta \gamma)}$ are operating on both the inelastic and thermal strains of the subcells.

It was shown by Dvorak and Benveniste (1992) and Dvorak (1992) that for any representative volume element under a uniform overall strain $\bar{\epsilon}$ and temperature change $\Delta T$, which contains a piecewise uniform distribution of thermal and inelastic fields (eigenstrains) associated with the applied loading $\bar{\epsilon}$, the averages of the local strain can be expressed in terms of the mechanical and eigenstrain concentration tensors. These tensors depend on the local elastic moduli, and on the shape and volume fraction of the phases and are therefore constant. Equation (66) is consistent with this representation of the average subcell strain, where the constant mechanical and eigenstrain concentration tensors are given by $\boldsymbol{A}^{(\alpha \beta \gamma)}$ and $D^{(\alpha \beta \gamma)}$, respectively.

Substitution of equation (66) into (8) yields

$$
\overline{\boldsymbol{\sigma}}^{(\alpha \beta \gamma)}=C^{(\alpha \beta \gamma)}\left[A^{(\alpha \beta \gamma)} \bar{\epsilon}+D^{(\alpha \beta \gamma)}\left(\epsilon_{s}^{I}+\epsilon_{s}^{T}\right)-\left(\bar{\epsilon}^{I(\alpha \beta \gamma)}+\epsilon^{T(\alpha \beta \gamma)}\right)\right]
$$

Consequently, in conjunction with eqn. (9), the following effective elastoplastic thermomechancial law of the composite can be established 


$$
\overline{\boldsymbol{\sigma}}=\boldsymbol{B}^{*}\left(\overline{\boldsymbol{\epsilon}}-\overline{\boldsymbol{\epsilon}}^{I}-\overline{\boldsymbol{\epsilon}}^{T}\right)
$$

where the effective elastic stiffness tensor, $\boldsymbol{B}^{*}$, of the composite is given by

$$
\boldsymbol{B}^{*}=\frac{1}{d h \ell} \sum_{\alpha=1}^{N_{\alpha}} \sum_{\beta=1}^{N_{\beta}} \sum_{\gamma=1}^{N_{\gamma}} d_{\alpha} h_{\beta} \ell_{\gamma} C^{(\alpha \beta \gamma)} A^{(\alpha \beta \gamma)}
$$

and the composite inelastic strain tensor is defined as

$$
\bar{\epsilon}^{I}=\frac{-B^{*-1}}{d h \ell} \sum_{\alpha=1}^{N_{\alpha}} \sum_{\beta=1}^{N_{\beta}} \sum_{\gamma=1}^{N_{\gamma}} d_{\alpha} h_{\beta} \ell_{\gamma} C^{(\alpha \beta \gamma)}\left(D^{(\alpha \beta \gamma)} \epsilon_{s}^{I}-\bar{\epsilon}^{I(\alpha \beta \gamma)}\right)
$$

and the average thermal strain tensor as

$$
\left.\overline{\boldsymbol{\epsilon}}^{T}=\frac{-B^{*-1}}{d h \ell} \sum_{\alpha=1}^{N_{\alpha}} \sum_{\beta=1}^{N_{\beta}} \sum_{\gamma=1}^{N_{\gamma}} d_{\alpha} h_{\beta} \ell_{\gamma} C^{(\alpha \beta \gamma)}\left(D^{(\alpha \beta \gamma)} \epsilon_{s}^{T}-\bar{\epsilon}^{T(\alpha \beta \gamma)}\right)\right)
$$

The effective coefficient of thermal expansion vector, $\boldsymbol{\alpha}^{*}$ of the composite, is given by

$$
\alpha^{*}=\bar{\epsilon}^{T} / \Delta T
$$

It can be numerically verified that the effective thermal stress vector $\Gamma^{*}=B^{*} \alpha^{*}$ is given in accordance with Levin (1967) result by

$$
\Gamma^{*}=\frac{1}{d h \ell} \sum_{\alpha=1}^{N_{\alpha}} \sum_{\beta=1}^{N_{\beta}} \sum_{\gamma=1}^{N_{\gamma}} d_{\alpha} h_{\beta} \ell_{\gamma} A^{T(\alpha \beta \gamma)} \Gamma^{(\alpha \beta \gamma)}
$$

where $\boldsymbol{A}^{T(\alpha \beta \gamma)}$ is the transpose of $\boldsymbol{A}^{(\alpha \beta \gamma)}$, and $\boldsymbol{\Gamma}^{(\alpha \beta \gamma)}$ is the thermal stress vector of the material filling subcell $(\alpha \beta \gamma)$. This implies that the generalized method of cells model is consistent. This consistency follows from the fact that when this model is employed to predict the effective coefficients of thermal expansion, it provides the same estimate obtained from Levin's result (73).

Like eqn. (73), it is possible (e.g. Dvorak (1992)) to establish the following estimate for the overall inelastic strain $\bar{\epsilon}^{I}$ of the composite

$$
\overline{\boldsymbol{\epsilon}}^{I}=\frac{1}{d h \ell} \sum_{\alpha=1}^{N_{\alpha}} \sum_{\beta=1}^{N_{\beta}} \sum_{\gamma=1}^{N_{\gamma}} d_{\alpha} h_{\beta} \ell_{\gamma} Q^{T(\alpha \beta \gamma)} \overline{\boldsymbol{\epsilon}}^{I(\alpha \beta \gamma)}
$$

where $\mathbf{Q}^{\mathrm{T}(\alpha \beta \gamma)}$ is the transpose of the mechanical concentration tensors, $Q^{(\alpha \beta \gamma)}$ that connect the stress $\overline{\boldsymbol{\sigma}}^{(\alpha \beta \gamma)}$ in the subcell to the total overall stress $\overline{\boldsymbol{\sigma}}$ of the composite subjected to homogeneous boundary conditions, i.e.

$$
\bar{\sigma}^{(\alpha \beta \gamma)}=Q^{(\alpha \beta \gamma)} \bar{\sigma}
$$

It can be easily verified that

$$
Q^{(\alpha \beta \gamma)}=C^{(\alpha \beta \gamma)} A^{(\alpha \beta \gamma)} B^{*-1}
$$


As in the thermal case, it can be numerically verified that the generalized cells model is consistent in the sense that the overall inelastic strain determined directly from eqn. (70), coincides with the values obtained by employing eqn. (74).

The micromechanically established overall thermo-inelastic constitutive law (68) is valid for any type of thermomechanical loading (i.e., any combination of normal, shear and thermal loadings). A significant advantage of this constitutive law stems from the fact that it does not rely on any symmetry conditions that may exist under certain types of applied loadings. Thus in the implementation of this law, the question as to whether such symmetry conditions exist or not is irrelevant.

\section{Conclusion}

A micromechanical theory is offered according to which an elastic-thermo-inelastic constitutive law which governs the overall behavior of short-fiber composites is established. This theory relies on the assumption that the composite possesses a periodic structure so that it is sufficient to analyze a repeating volume element. This repeating volume element (or cell) can include an arbitrary number of subcells, so that multiphased composites containing short fibers and arranged in various configurations (e.g. square, hexagonal, or square diagonal packing) can be considered.

The established constitutive law is based on the principles of continuum mechanics where microequilibrium and continuity of displacements and tractions at the various interfaces are ensured in an averaged sense. The key ingredient in the construction of this macro constitutive law, is the establishment of the appropriate concentration tensors, $\boldsymbol{A}^{(\alpha \beta \gamma)}$ and $D^{(\alpha \beta \gamma)}$ at the microlevel. Given these concentration tensors, the average stress and strain micro field variables in the subcell can then be expressed in terms of the uniform overall strain and the subcell inelastic and thermal strains, see equations (66) and (67). The macromechanical law is then established by applying a homogenization (or averaging) procedure, see equations ( 7 ) and (9), to the microscopic thermo-inelastic constitutive equations.

The established analytic constitutive law can be readily applied to investigate the behavior of various types of composites given knowledge of the behavior of the individual phases. Numerous advantages can be stated regarding the current macro/micro constitutive laws as compared to the other numerical micromechanical approaches in the literature, e.g. the finite element unit cell approach. One advantage is the fact that any type of simple or combined loading (multiaxial state of stress) can be applied irrespective of whether symmetry exist or not, as well as without resorting to different boundary condition application strategies as in the case of the finite element unit cell procedure. Another, advantage concerns the availability of an analytical expression representing the macro elastic-thermo-inelastic constitutive law, thus ensuring an economy of memory when implementing this formulation into a structural finite element analysis code. Furthermore, this formulation has been shown to predict accurate macro behavior given only a few number of subcells, within the repeating cell (see Paley and Aboudi (1991), and Arnold et al.(1993)). Whereas, if one employs the finite element unit cell procedure, a significant number of finite elements are required within a given repeating cell to obtain the same level of accuracy as with the present formulation. As a result it is possible to utilize the presented constitutive law to efficiently analyze metal matrix 
composite structures subjected to complex thermomechanical loadings. This is particularly important when analyzing realistic structural components, since different loading conditions exist throughout the structure, thus necessitating the application of the macromechanical equations repeatedly at these locations.

\section{Acknowlegement}

The author gratefully acknowledges the support provided by NASA -Lewis Research Center through grant NAG 3-1377. Also special thanks go to Dr. S.M. Arnold, the technical monitor, for his encouragement and discussions regarding this work and assistance with the preparation of this report.

\section{References}

Aboudi, J., Mechanics of Composite Materials - A Unified Micromechanical Approach. Elsevier, Amsterdam, 1991.

Aboudi, J., Constitutive Behavior of Multiphase Metal Matrix Composites with Interfacial Damage by the Generalized Cell Model, in : G.Z. Voyiadjis, ed., Damage in Composite Materials, Elsevier, Amsterdam, p. 3, 1993.

Aboudi, J. and Pindera, Micromechanics of Metal Matrix Composites Using the Generalized Method of Cells Model (GMC) User's Guide. NASA CR 190756, 1992.

Arnold, S.M., Wilt, T.E., Saleeb, A.F., and Castelli, M.G., "An Investigation of Macro and Micromechanical Approaches for a Model MMC System", NASA CP 19117, Vol. II, pp. (52.1)-(52.12), 1993.

Dvorak, G.J., Transformation Field Analysis of Inelastic Composite Materials. proc. R. Soc. Lond (A) 437, 311-327, 1992.

Dvorak, G.J. and Y. Benveniste, On Transformation Strains and Uniform Fields in Multiphase Elastic Media. Proc. R. Soc. Lond. (A), 437, 291-310, 1992.

Levin, V.M., On the Coefficients of Thermal Expansion of Heterogeneous Materials, Mech. of Solids, $\underline{2}, 58-61,1967$.

Paley, M. and Aboudi, J. (1991), Micromechanical Analysis of Composites by the Generalized Cell Model. Mech. of Materials, 14, 127-139, 1992.

Suquet, P.M., Local and Global Aspects in the Mathematical Theory of Plasticity, in: A. Sawezuk and G. Bianchi, Eds., Plasticity Today, Elsevier, Amsterdam, p. 279, 1985. 


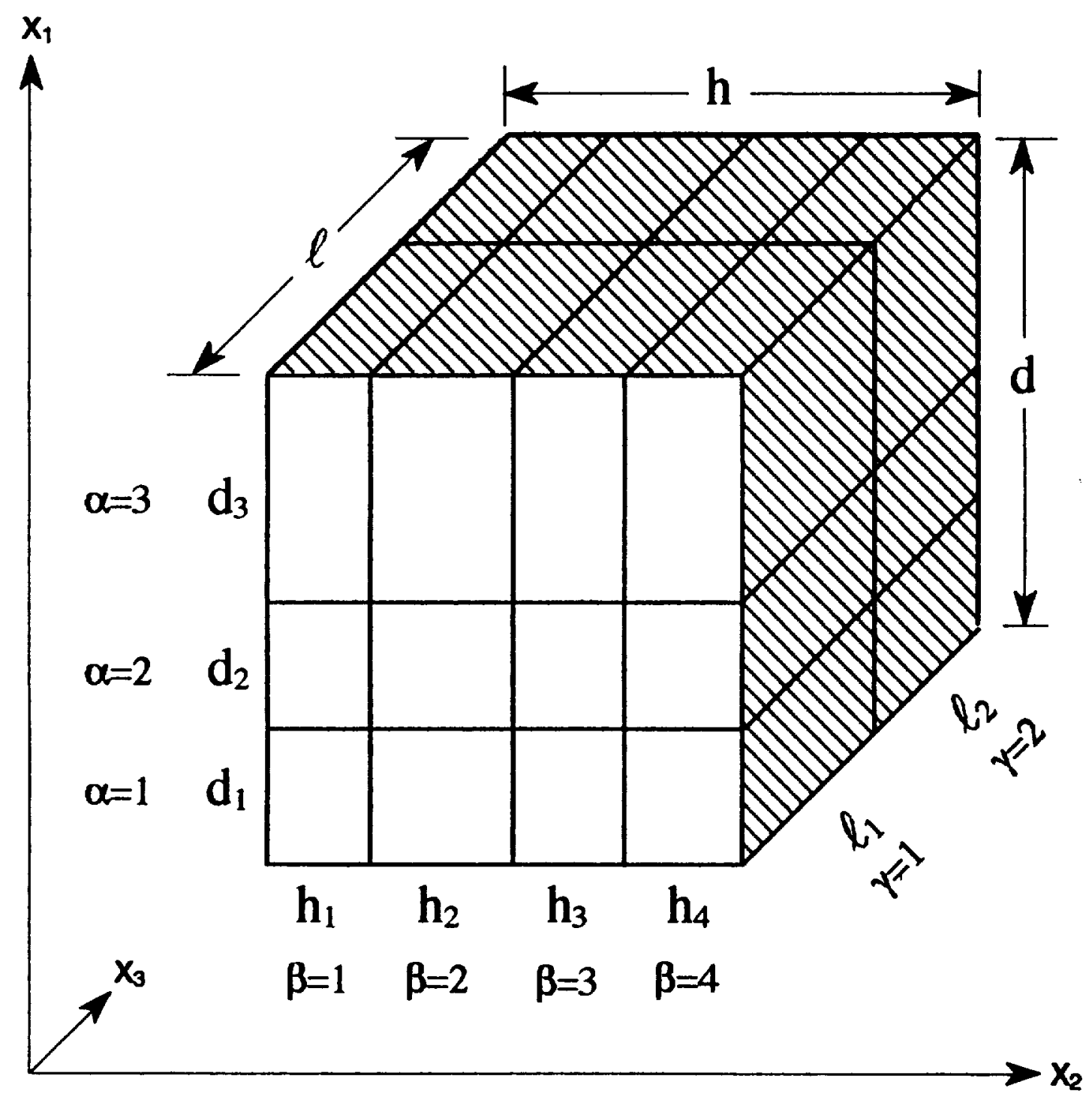

Fig. 1. (a) A repeating volume element that consists of $N_{\alpha}=3, N_{\beta}=4$ and $N_{\gamma}=2$ subcells. 


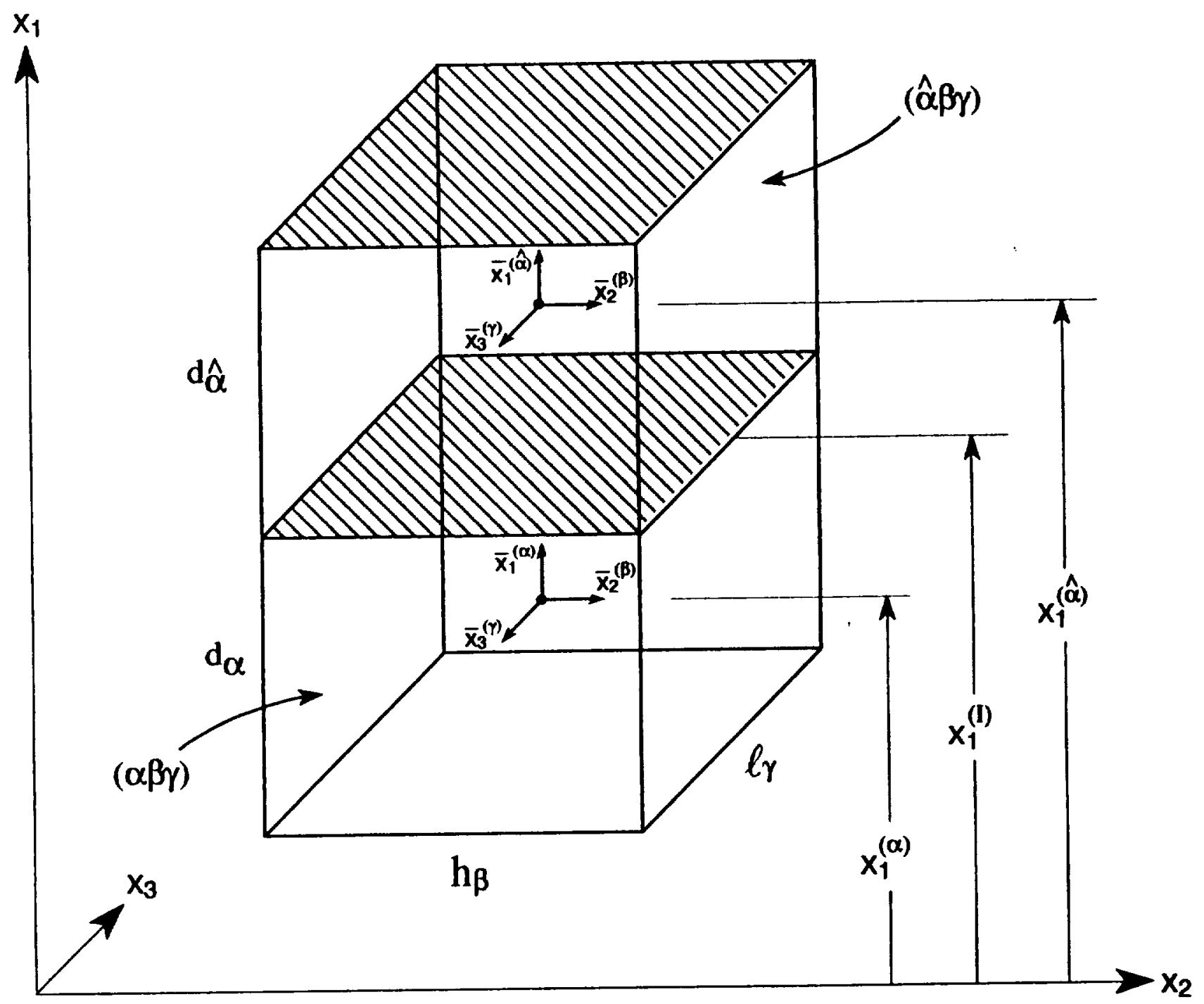

Fig. 2. The local coordinates, and locations of interface and centerlines of typical two neighboring subcells. 


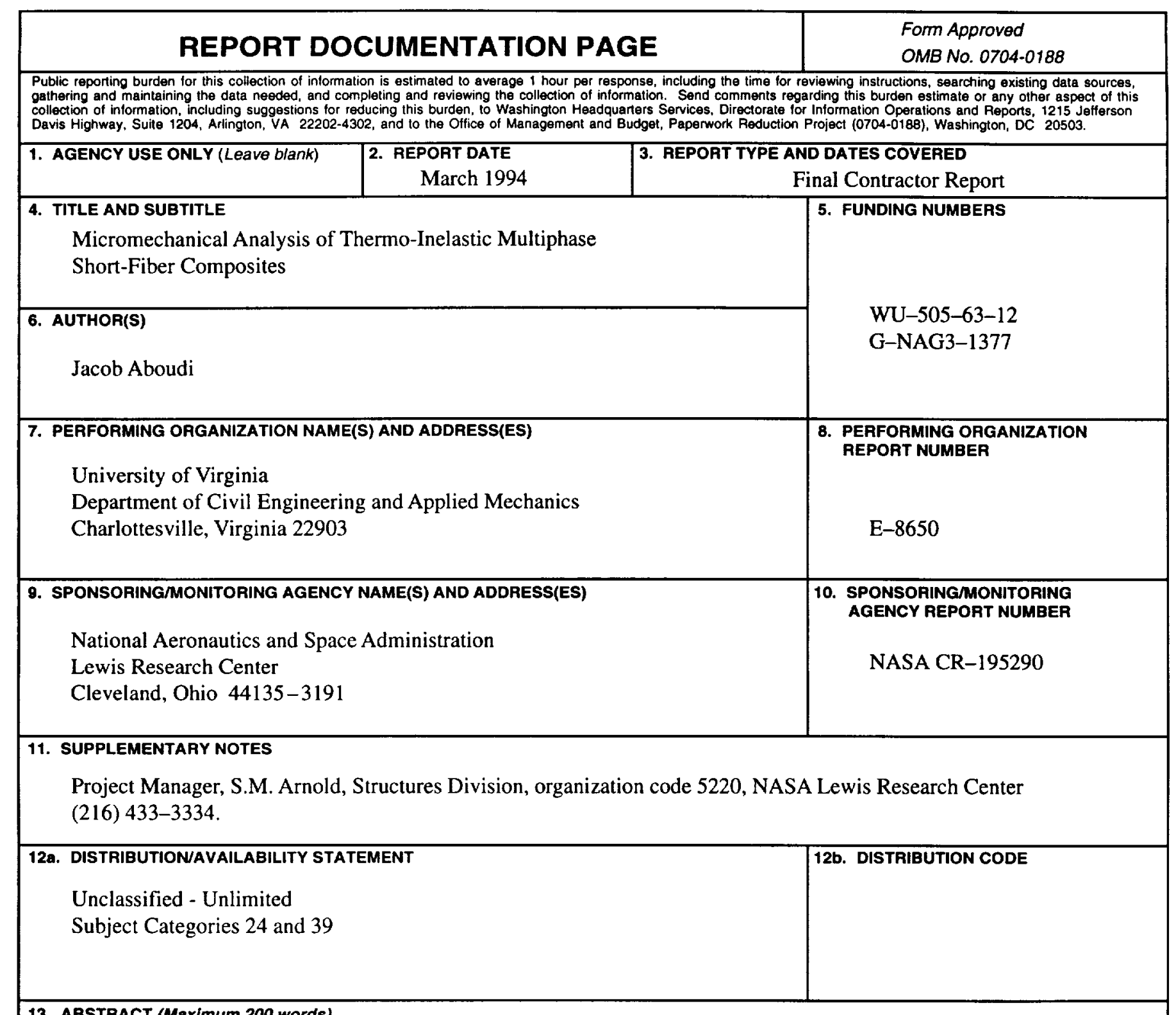

13. ABSTRACT (Maximum 200 words)

A micromechanical formulation is presented for the prediction of the overall thermo-inelastic behavior of multiphase composites which consist of short fibers. The analysis is an extension of the generalized method of cells that was previously derived for inelastic composites with continuous fibers, and the reliability of which was critically examined in several situations. The resulting three-dimensional formulation is extremely general, wherein the analysis of thermoinelastic composites with continuous fibers as well as particulate and porous inelastic materials are merely special cases.

14. SUBJECT TERMS

Discontinuous composites: Micromechanics: Viscoplasticity: Nonisothermal

15. NUMBER OF PAGES

19

16. PAICE CODE

$\mathrm{A} 03$

17. SECURITY CLASSIFICATION OF REPORT

Unclassified

18. SECURITY CLASSIFICATION OF THIS PAGE

Unclassified

19. SECURITY CLASSIFICATION OF ABSTRACT Unclassified 